\title{
Conhecimento, consumo e acesso à contracepção de emergência entre mulheres universitárias no sul do Estado de Santa Catarina
}

\author{
Awareness, consumption and access \\ to emergency contraception among female university students \\ in the south of the State of Santa Catarina
}

Graziela Modolon Alano ${ }^{1}$
Laise Nunes Costa $^{1}$
Luziane Righeto Miranda $^{1}$
Dayani Galato ${ }^{1}$

${ }^{1}$ Núcleo de Atenção Farmacêutica e Estudos da Utilização de Medicamentos (NAFEUM), Curso de

Farmácia, Universidade do Sul de Santa Catarina (UNISUL). Av. José Acácio Moreira 787, Dehon. 88704-900 Tubarão SC. graziela.alano@unisul.br

\begin{abstract}
The scope of this study was to investigate knowledge about and access to emergency contraception among female university students in the south of the State of Santa Catarina. A crosssectional observational study was conducted between September and October 2008, in which a questionnaire was given to 360 women aged 18 to 45 years. Most belonged to the B2 economic class or higher (74.2\%) and 79.4\% of them had already had intercourse. Of these, $48.6 \%$ had used emergency contraception, though this was more frequent among younger women. Emergency contraception was used 2.4 times, and $87.1 \%$ had used contraceptive methods within 24 hours after intercourse. Adverse reactions were reported by 20.9\%, the most common being menstrual cycle changes (44.8\%) and nausea (44.8\%). The reasons for using emergency contraception included the lack of condom use (44.6\%) and condom rupture (39.6\%). Of the women interviewed, 15\% either failed to answer the question on STD prevention or answered incorrectly, while $97.8 \%$ denied having changed the method used routinely. Only 2.9\% of the participants had acquired the medication via prescription, and $35.3 \%$ received guidance on application of the medication at the time of purchase.
\end{abstract}

Key words Emergency contraception (use), Morning-after pill, Women, Students, Sexually Transmitted Disease
Resumo A contracepção de emergência tem sido utilizada na prevenção da gravidez não planejada. Este estudo objetivou investigar o conhecimento, o consumo e o acesso desse método entre mulheres universitárias do Sul do Estado de Santa Catarina. Trata-se de estudo observacional, transversal, aplicando-se formulário de setembro a outubro de 2008. Participaram 360 mulheres entre 18 e 45 anos, a maioria da classe econômica B2 ou superior $(74,2 \%)$, onde $79,4 \%$ já haviam mantido relação sexual. Destas, 48,6\% haviam utilizado a contracepção de emergência, sendo mais frequente quanto menor a idade. O método foi usado em média 2,4 vezes e 87,1\% administraram-no até 24 horas após a relação sexual. Foram referidas reações adversas por $20,9 \%$ das usuárias, sendo as mais comuns, alterações menstruais (44,8\%) e náuseas $(44,8 \%)$. Os principais motivos para o uso do método foram: não uso do preservativo $(44,6 \%) e$ rompimento do mesmo (39,6\%). Das entrevistadas, 15,0\% não souberam responder ou responderam de modo errado à questão sobre a não prevenção de Doença Sexualmente Transmissível pelo método e 97,8\% negaram ter alterado o método utilizado rotineiramente. Apenas 2,9\% das mulheres adquiriram o medicamento mediante prescrição médica e 35,3\% receberam orientações no momento da compra.

Palavras-chave Contracepção de emergência (utilização), Pílula do dia seguinte, Mulheres, Universitárias, Doença Sexualmente Transmissivvel 


\section{Introdução}

A Contracepção de Emergência (CE) consiste na ingestão de uma substância hormonal, o levonorgestrel isolado, que deve ser tomada até 120 horas após uma relação sexual desprotegida sendo, no entanto, mais eficaz quanto mais precocemente for instituída ${ }^{1}$. Também chamada de pílula do dia seguinte pode ser usada em situações com risco de gravidez como no uso inadequado de método anticoncepcional regular, no rompimento de preservativo ou após um ato de violência sexual ${ }^{2}$.

O método de contracepção de emergência deve ser empregado de modo ocasional e nas situações específicas, pois, além de não fornecer proteção contra Doença Sexualmente Transmissível (DST), existem outros métodos contraceptivos mais eficazes para uso contínuo ${ }^{3}$.

O mecanismo de ação deste método dependerá do momento do ciclo menstrual em que for administrado. O levonorgestrel pode atuar impedindo ou retardando a ovulação devido à alteração da motilidade tubária, por inversão do peristaltismo e do batimento das fímbrias, e alteração da motilidade dos espermatozóides, pelo aumento do $\mathrm{pH}$ do fluido uterino e da viscosidade do muco cervical. Alguns autores defendem que a CE também pode impedir a fecundação interferindo no preparo do endométrio ${ }^{4}$. Porém, segundo o Ministério da Saúde e Costa et al. ${ }^{5}$, não há quaisquer evidências científicas de que a contracepção de emergência exerça efeitos após a fecundação ou que implique a eliminação precoce do embrião.

No Brasil, o método contraceptivo de emergência é regulamentado pelo Ministério da Saúde e aprovado pela vigilância sanitária estando disponível, comercialmente, mediante prescrição médica. Além disso, a CE está incluída pelo Ministério da Saúde nas normas técnicas de Planejamento Familiar (1996) e Violência Sexual $(1998)^{4}$.

Alguns autores declaram que o contraceptivo de emergência ainda apresenta baixa incidência de uso $^{3}$ apontando barreiras ao acesso, como a falta de informação, aspectos culturais e morais, exigência de prescrição médica, preço dos produtos, além de preocupação excessiva dos profissionais de saúde quanto a possibilidade de influenciar sobre o uso regular de métodos anticoncepcionais, como o preservativo ${ }^{5}$. Costa et al. ${ }^{5}$ destacam que, faltam estudos no Brasil sobre a prevalência de uso do método e sua influência sobre a adoção de métodos de contracepção de uso con- tínuo, bem como o conhecimento pelas mulheres da não prevenção de DST pelo método.

Assim, o objetivo do estudo foi investigar a utilização da contracepção de emergência, composta por levonorgestrel isolado, por mulheres universitárias, com o propósito de fornecer subsídios para a adoção de estratégias que promovam o uso racional desse produto.

\section{Métodos}

Trata-se de um estudo observacional quantitativo, do tipo transversal, aplicando-se um formulário autoaplicativo, no período entre setembro e outubro de 2008, para obtenção de informação sobre a utilização do método em estudo, bem como conhecimentos sobre o mesmo.

A população da pesquisa envolveu mulheres universitárias, do primeiro ano de graduação de uma instituição de ensino superior do Sul do Estado de Santa Catarina, localizada a 130 quilômetros da capital do Estado, Florianópolis. A universidade é composta por 44 cursos que estão organizados em quatro áreas: gestão e jurídica, tecnológica, saúde, comunicação e educação.

Adotando-se o mesmo procedimento de amostragem desenvolvido por Souza et al. ${ }^{6}$, inicialmente foi realizado o sorteio de 12 cursos, sendo a amostra composta por três representantes de cada uma das áreas apresentadas anteriormente, estes possuíam em torno de $480 \mathrm{mu}$ lheres no período investigado.

O critério de inclusão foi cursar o primeiro ano da graduação e pertencer aos cursos sorteados e estar presente na sala de aula no memento da aplicação do instrumento e o de exclusão, possuir idade inferior a 18 anos e não aceitar participar da pesquisa. Definido os critérios, a amostra totalizou 360 mulheres a serem investigadas.

$\mathrm{O}$ instrumento utilizado foi dividido em três partes, na primeira foram solicitadas informações sobre o perfil das entrevistadas como idade, curso e nível socioeconômico ${ }^{7}$; na segunda parte, foi investigado o conhecimento sobre a não prevenção de DST pela contracepção de emergência e sobre sexualidade (idade da primeira relação sexual). A terceira parte do instrumento foi aplicada apenas àquelas mulheres que já tinham tido relação sexual, sendo, neste caso, questionado o tipo de parceiro sexual atual, se conhece o método e se houve utilização da CE em alguma situação. Na resposta afirmativa para esta última questão, foram coletadas outras informações, incluindo: frequência de utilização, me- 
nor intervalo de tempo entre duas situações de uso, motivo que levou a adoção do método, tempo entre a relação desprotegida e a administração da CE, reações adversas, alteração do método contraceptivo de uso regular após a disponibilização comercial da CE, ocorrência de gravidez, apresentação de prescrição médica para a aquisição e orientações recebidas no momento da dispensação.

Foi realizado um pré-teste do instrumento envolvendo 20 mulheres da universidade em estudo, a fim de avaliar a legibilidade e aplicabilidade. Os dados desta etapa não foram incluídos na pesquisa.

Para a coleta de dados, inicialmente foi realizada a apresentação do estudo às coordenações dos cursos sorteados e realizado um levantamento das mulheres matriculadas no primeiro ano. O formulário foi então aplicado, dentro da sala de aula do curso sorteado, às universitárias que aceitaram participar do estudo ao assinar o Termo de Consentimento Livre e Esclarecido. O tempo para a aplicação foi entre 10 a 20 minutos para cada turma e ocorreu durante os meses de setembro e outubro de 2008.

A partir dos dados coletados foi criado um banco de dados no programa EpiData ${ }^{8}$ versão $3.1^{\circledast}$ e analisados no EpiInfo ${ }^{9} 6.0^{\varpi}$. As variáveis nominais foram apresentadas em números absolutos e proporções e as variáveis numéricas em medidas de tendência central e dispersão. Para analisar a associação entre a variável de desfecho (uso de contracepção e emergência, frequência de uso e conhecimento sobre a prevenção de DST) e as variáveis de exposição (perfil das entrevistadas) foi adotado o teste do qui-quadrado, adotou-se $\mathrm{p}<0,05$ como significativo.

O projeto foi aprovado pelo Comitê de Ética em Pesquisa da universidade em estudo.

\section{Resultados}

Participaram do estudo 360 mulheres com idade média de 21,2 ( \pm 4,94) anos, variando entre 18 e 45 anos, pertencentes na maioria a classe B2 ou superior $^{7}$ e aos cursos de Direito (22,8\%), Nutrição $(14,4 \%)$ e Ciências Contábeis (13,3\%). Destaca-se que no curso de Química Industrial havia apenas uma mulher nas turmas e no curso Sistema de Informação não havia mulheres. A maioria das mulheres já havia mantido relações sexuais $(79,4 \%)$, sendo a idade média da primeira relação de 17,1 ( $\pm 1,71)$ anos. Destas, 85\% afirmaram possuir parceiro atualmente e de modo fixo
97,2\%. Das entrevistadas 85\% declararam saber que o método não previne DST e número reduzido de mulheres declarou não ter conhecimento da existência do método (1\%) (Tabela 1).

Parcela considerável das entrevistadas havia feito uso da contracepção de emergência $(48,6 \%)$, sendo usado $2,4( \pm 1,9)$ vezes, obtendo-se variação entre 1 a 16 vezes de utilização deste método. De 88 mulheres que informaram o menor intervalo de tempo entre duas situações de uso 69,3\% referiram utilizar em período inferior a seis meses, sendo que o período mais prevalente foi de um mês com 25,0\%. Os motivos para a adoção do método mais frequente foram o não uso do preservativo em $44,6 \%$ ou rompimento do mesmo com 39,6\% (Tabela 2).

Tabela 1. Características relacionadas ao nível socioeconômico, ao curso, a sexualidade e o conhecimento sobre a Contracepção de Emergência pelas mulheres entrevistadas.

\begin{tabular}{|c|c|c|}
\hline Características & $\mathbf{n}$ & $\%$ \\
\hline \multicolumn{3}{|l|}{ Nível socioeconômico $(\mathrm{n}=360)$} \\
\hline A 1 & 3 & 0,8 \\
\hline A2 & 36 & 10,0 \\
\hline B1 & 91 & 25,3 \\
\hline B2 & 137 & 38,1 \\
\hline $\mathrm{C} 1$ & 61 & 16,9 \\
\hline $\mathrm{C} 2$ & 24 & 6,7 \\
\hline $\mathrm{D}$ & 7 & 1,9 \\
\hline $\mathrm{E}$ & 1 & 0,3 \\
\hline \multicolumn{3}{|l|}{ Curso de graduação $(\mathrm{n}=360)$} \\
\hline Gestão e jurídica & 144 & 40,0 \\
\hline Ciências Contábeis & 48 & 13,3 \\
\hline Direito & 82 & 22,8 \\
\hline Técnico de marketing e vendas & 14 & 3,9 \\
\hline Tecnológica & 42 & 11,7 \\
\hline Química industrial & 1 & 0,3 \\
\hline Técnico em modas & 28 & 7,8 \\
\hline Arquitetura e urbanismo & 13 & 3,6 \\
\hline Saúde & 96 & 26,6 \\
\hline Farmácia & 22 & 6,1 \\
\hline Enfermagem & 22 & 6,1 \\
\hline Nutrição & 52 & 14,4 \\
\hline Comunicação e educação & 78 & 21,7 \\
\hline Letras português & 39 & 10,8 \\
\hline Pedagogia & 39 & 10,8 \\
\hline Sistema de informação & 0 & 0,0 \\
\hline Já mantiveram relações sexuais $(\mathrm{n}=358)$ & 286 & 79,4 \\
\hline Possuem parceiro sexual atualmente $(\mathrm{n}=246)$ & 211 & 85,7 \\
\hline \multicolumn{3}{|l|}{ Tipo de parceiro $(\mathrm{n}=246)$} \\
\hline Fixo & 239 & 97,2 \\
\hline Não fixo & 7 & 2,8 \\
\hline $\begin{array}{l}\text { Sabem que CE não previne doença sexualmente } \\
\text { transmissível/HIV }(\mathrm{n}=358)\end{array}$ & 304 & 85,0 \\
\hline Sabem da existência da CE $(\mathrm{n}=288)$ & 285 & 99,0 \\
\hline
\end{tabular}


Uma das universitárias, embora tenha apontado no formulário como motivo o não uso do preservativo, descreveu ter feito uso devido a um ato de violência sexual sofrido pela mesma.

A maioria das universitárias administrou a contracepção de emergência nas primeiras $24 \mathrm{~h}$ após o ato sexual $(87,1 \%)$, tendo como reação adversa mais frequente a alteração do ciclo menstrual $(44,8 \%)$ e náuseas $(44,8 \%)$. Parcela considerável das entrevistadas $(97,8 \%)$ declarou não haver ocorrido modificação no uso regular do método contraceptivo adotado por elas até o momento da pesquisa (Tabela 2).

Quando questionadas a respeito da ocorrência de gravidez após a utilização de CE $(\mathrm{n}=139)$, uma afirmou ter engravidado. Esta relatou ter utilizado o método várias vezes (não declarando número exato) com menor intervalo entre duas situações de uso de três meses. O motivo apontado para a utilização deste método foi o rompimento do preservativo e em relação às reações adversas a universitária relatou ter apresentado náuseas, vômito, sangramento, cólicas, cefaleia e alterações no ciclo menstrual.

A maioria das mulheres adquiriu o método sem apresentação da prescrição médica $(97,1 \%)$ e $35,3 \%$ afirmaram ter recebido alguma informação no momento da compra, sendo mais fre-

Tabela 2. Dados sobre a utilização da Contracepção de Emergência pelas mulheres entrevistadas.

\begin{tabular}{lrr}
\hline Dados sobre a Contracepção de Emergência & n & \multicolumn{1}{c}{$\%$} \\
\hline Utilizaram a CE $(\mathrm{n}=288)$ & 140 & 48,6 \\
Motivos de uso $(\mathrm{n}=139)$ & & \\
Não uso do preservativo & 62 & 44,6 \\
Rompimento do preservativo & 55 & 39,6 \\
Uso incorreto do anticoncepcional oral & 35 & 25,2 \\
Tempo de administração após a relação (n=140) & & \\
Até 24h & 122 & 87,1 \\
24 a 48h & 8 & 5,7 \\
48 a 72h & 6 & 4,3 \\
Não souberam responder & 4 & 2,9 \\
Apresentaram reações adversas após uso da CE & 60 & 20,9 \\
Alteração do ciclo menstrual & 27 & 44,8 \\
Sangramento & 17 & 27,6 \\
Náuseas & 27 & 44,8 \\
Vômito & 8 & 13,8 \\
Cefaléia & 6 & 10,3 \\
Houve modificação do método contraceptivo & & \\
regular após a disponibilização do CE (n = 138) & & \\
Não & 135 & 97,8 \\
Sim & 3 & 2,2 \\
\hline
\end{tabular}

quente a orientação sobre o intervalo entre doses na medicação com doses divididas $(75,5 \%)$, conforme descrito na Tabela 3.

Constatou-se nesse estudo que, quanto menor a idade da universitária maior foi a prevalência de utilização do método $(\mathrm{p}=0,02961)$. Não foi encontrada associação significativa entre usar o método de contracepção de emergência, assim como a frequência de uso desse método, e as demais variáveis relacionadas ao perfil das entrevistadas, conforme apresentado na Tabela 4 .

\section{Discussão}

$\mathrm{Na}$ atualidade, a vida sexual tem sido iniciada cada vez mais cedo, como evidenciado neste estudo e por Costa et al. ${ }^{5}$, onde mulheres iniciaram a partir dos 11 anos. De acordo com Taquette et al. ${ }^{10} \mathrm{o}$ cedo início da primeira relação sexual junto ao maior número de parceiros, pode contribuir para aumentar a ocorrência de DST. Nesse estudo, constatou-se que a maioria das mulheres possui parceiro fixo. Entretanto, não definiu o que seria considerado como parceiro fixo, o que pode ter prejudicado a análise desse resultado.

Parcela considerável das mulheres (15\%) não demonstrou conhecimento sobre o fato da contracepção de emergência não prevenir DST, dado esse preocupante visto que se trata de jovens inseridas no meio acadêmico e que, por isso, têm um acesso facilitado às informações. Em trabalhos publicados por Azevedo e Abdo ${ }^{11}$ e Souza et al. ${ }^{6}$, foi demonstrado que o nível de conhecimento sobre prevenção de DST ainda permanece baixo.

Costa et al. ${ }^{5}$ afirmam que o acesso à contracepção de emergência pode ser dificultado pela exigência de prescrição médica. Entretanto, ob-

Tabela 3. Dados sobre a aquisição da contracepção de emergência pelas mulheres entrevistadas.

\begin{tabular}{lcc}
\hline \multicolumn{1}{c}{ Dados sobre a aquisição do CE } & n & \% \\
\hline $\begin{array}{l}\text { Compraram a CE mediante prescrição } \\
\text { médica }(\mathrm{n}=139)\end{array}$ & 4 & 2,9 \\
Receberam orientações na compra da & 49 & 35,3 \\
CE $(\mathrm{n}=139)$ & & \\
Informações repassadas na compra da & & \\
CE (n = 49) & & \\
$\quad \begin{array}{l}\text { Intervalo entre doses na medicação } \\
\text { com doses divididas }\end{array}$ & 37 & 75,5 \\
$\quad$ Tempo de uso após a relação sexual & 28 & 57,1 \\
$\quad$ Efeitos colaterais & 9 & 18,4 \\
\hline
\end{tabular}


Tabela 4. Associação entre variáveis de desfecho e de exposição.

\begin{tabular}{lc}
\multicolumn{1}{c}{ Variáveis de Desfecho e de Exposição } & Valor de significância (p) \\
\hline Utilizar a CE & 0,030 \\
Idade & 0,735 \\
Nível socioeconômico & 0,088 \\
Pertencer a cursos de graduação na área da saúde & \\
Conhecimento das mulheres sobre a não prevenção de DST/HIV pela CE & 0,909 \\
Idade & 0,375 \\
Nível socioeconômico & 0,713 \\
Pertencer a cursos de graduação na área da saúde & 0,880 \\
Receber informações no momento da aquisição do medicamento & 0,076 \\
Ter iniciado a vida sexual & \\
Frequência de uso da CE & 0,758 \\
Idade & 0,705 \\
Nível socioeconômico & 0,492 \\
Pertencer a cursos de graduação na área da saúde & 0,995 \\
Ocorrência de reações adversas & \\
\hline
\end{tabular}

serva-se que essa exigência não é cumprida, pois milhares de pessoas adquirem facilmente o produto nas farmácias como constatado nos relatos das universitárias, onde somente $2,9 \%$ relataram apresentar a prescrição no momento da aquisição; bem como no estudo de Borges et al. ${ }^{12}$ onde apenas $4,3 \%$ das entrevistadas apresentaram prescrição.

$\mathrm{O}$ acesso à contracepção de emergência ainda está sendo discutido em diversos países, sendo que em alguns deles o produto pode ser adquirido sem prescrição, como nos EUA desde 2006. Estudo realizado ${ }^{13}$ nesse país, não demonstrou diferença na taxa de gravidez não planejada antes ou depois da CE tornar-se um produto sem exigência de prescrição. Os autores destacam que, a falta de conhecimento e entendimento sobre a CE pode ser considerada a maior barreira para o uso apropriado desse método na população.

Atualmente, o método de contracepção de emergência faz parte das Normas de Planejamento Familiar como forma de garantir o direito de controle de fecundidade fornecido às mulheres ou ao casal, porém sua disponibilização no sistema de saúde ainda não é uma realidade em muitas localidades. De acordo Souza e Brandão ${ }^{14}$, existe uma resistência por parte dos serviços públicos para disponibilizar esse método devido ao senso comum de que a CE levaria os usuários, principalmente adolescentes, a abandonar outros métodos contraceptivos de uso regular, inclusive preservativo, fato não confirmado por estudos realizados, inclusive este mesmo.
Parcela considerável declarou saber da existência da contracepção de emergência (99\%) como um método pós-coito para se evitar uma gravidez não planejada, sendo que destas 48,6\% haviam feito uso do método. Este dado demonstra que a informação sobre a existência deste método abrange a maioria das mulheres universitárias em estudo e foi evidenciado consumo elevado do mesmo pelas entrevistadas. Estudo de Borges et al. ${ }^{12}$ também encontrou valor semelhante em universitárias de São Paulo, onde $50,4 \%$ haviam feito uso desse método.

Os resultados deste estudo mostram que o uso da CE foi bastante alto entre as universitárias, considerando os dados nacionais levantados pela Pesquisa Nacional de Demografia e Saúde da Criança e da Mulher (PNDS) de 2006, cuja prevalência maior foi encontrada na faixa etária compreendida entre 20 e 24 anos (18,5\%), seguida do grupo de mulheres com idade entre 25 e 29 anos (16,9\%), e os menores índices estão entre os adolescentes $(10,4 \%)^{15}$. Percebe-se que, os valores da prevalência de uso do método podem variar de acordo com a faixa etária pesquisada e o delineamento da pesquisa, como mostra o estudo de Araújo e Costa ${ }^{16}$ envolvendo adolescentes de 14-19 anos de idade, no Estado de Pernambuco, onde houve uso por $27,8 \%$ dos estudantes; e o estudo de Figueiredo ${ }^{17}$ que, também encontrou baixa prevalência de uso da CE por mulheres com HIV/AIDS (2,7\%). Portanto, devese ter cautela ao realizar o comparativo entre diversos estudos, para se evitar uma conclusão 
precipitada sobre o perfil de consumo desse método no país.

O método foi usado em média 2,4 vezes pelas universitárias até o momento do estudo, assim como encontrado em outras pesquisas no Brasil $^{12}$ e na Espanha ${ }^{18}$. Quanto ao intervalo de tempo entre duas situações na qual tiveram que usar a CE, 25,0\% referiu ter utilizado duas vezes em um único mês. Cabe ressaltar que o método de CE deve ser empregado de modo ocasional e em situações específicas, podendo ser considerado pouco eficaz se utilizado repetidamente (a curtos intervalos), pois é o menos eficaz entre os métodos modernos para a contracepção rotineira ${ }^{2-4}$.

Quanto aos motivos para o uso do método encontrou-se o não uso do preservativo, o rompimento do mesmo e o uso incorreto de anticoncepcionais orais. Estudo de Borges et al..$^{12}$ encontrou resultado semelhante. Sendo o preservativo o método mais adequado para prevenção de DST/ AIDS torna-se importante a realização de investigações aprofundadas sobre esse resultado.

Ressalta-se que, neste estudo, a maioria das universitárias utilizou a contracepção de emergência nas primeiras 24 horas após a relação sexual, o que pode ser considerado satisfatório, pois quanto mais precocemente for utilizado o método, maior será sua efetividade ${ }^{4}$. Resultados semelhantes quanto ao período de tempo entre a relação sexual e o uso do método foram obtidos nos estudos de Costa et al. ${ }^{5}$.

Das mulheres que apresentaram reação adversa, os mesmos corroboram com a literatura ${ }^{5}$. Alterações significantes, mas transitórias, no ciclo menstrual são comuns após o uso do método, como confirmado por Gainer et al. ${ }^{19}$. Porém, apesar de em outros estudos a cefaleia ser um dos sintomas mais citados ${ }^{3,5}$, nessa pesquisa apresentou pouca frequência. Wannmacher ${ }^{3}$ afirma que cefaleia é um sintoma mais comum no caso de dose única de levonorgestrel em comparação as doses fracionadas (12 em 12 horas), fato este que não foi investigado no presente estudo e poderia ser um dos motivos da baixa incidência dessa reação adversa no mesmo.

A adoção de um método contraceptivo de modo regular, após a disponibilização comercial da CE, não foi alterada segundo $97,8 \%$ das universitárias. Outros estudos têm demonstrado resultados semelhantes ${ }^{3,5}$.

Pequena parcela das universitárias relatou ter recebido orientações sobre a CE no momento da aquisição do produto na farmácia. Dessas, a informação sobre intervalo entre as doses, quando o produto foi empregado em doses divididas, foi a mais frequente. Esse dado salienta a necessidade de melhor atuação do farmacêutico na orientação das mulheres que procuram o método de $\mathrm{CE}$, garantindo o uso correto, bem como, para a prevenção de DST/HIV estimulando o uso do preservativo, assim como destacado por Bastos et $a .^{20}$. A orientação de prevenção às DST/HIV poderia ser realizada na farmácia, nos casos de busca de informação e utilização de métodos contraceptivos de emergência e de uso regular, como um critério de qualidade no atendimento. Considera-se, desse modo, importante que profissionais do setor farmacêutico tenham formação específica para que possam exercer adequadamente a função de educadores de saúde, inerente à atividade que desempenham, e que atuem no sentido de estabelecer referência entre estabelecimentos farmacêuticos e o Sistema Único de Saúde (SUS) ${ }^{20}$.

Realizando-se a análise estatística, constatouse que as mulheres mais jovens tendem a maior utilização do método. O que poderia ser justificada pelo medo de uma gravidez não planejada, por serem jovens para assumir a responsabilidade de ter filhos, somado ao fato de serem, muitas vezes, totalmente dependentes dos pais ou mesmo por não terem uma vida economicamente estável.

O planejamento familiar torna-se essencial para se evitar uma gravidez não planejada, sendo as mulheres com nível socioeconômico mais baixo e menor nível de escolaridade, a parcela mais atingida por esse tipo de situação, pois a maioria não possui acesso às informações e aos métodos contraceptivos adequados ${ }^{15}$. Como uma das estratégias apontadas para amenizar este problema, é indispensável que o acesso à contracepção de emergência e aos demais métodos de contracepção e proteção e tratamento para DST e HIV/ Aids esteja garantido na rede pública de saúde ${ }^{20}$.

O estudo apresentou limitações advindas do fato de obter informações retrospectivas, o que devido à sua natureza, poderia estar sujeito ao erro de memória. Porém, tal situação não invalida os resultados obtidos permitindo que o estudo forneça subsídios para a avaliação do perfil de uso deste medicamento pelas universitárias.

São destacados os resultados encontrados neste estudo, principalmente em relação ao elevado uso de contracepção de emergência, em sua maioria motivada pelo não uso ou rompimento do preservativo; o fato do método ter sido adquirido sem apresentação da prescrição médica; que parte das universitárias não tem conhecimento sobre a não prevenção de DST/HIV pelo 
método; e, que a disponibilização desse produto não promoveu alteração no uso do método de contracepção regular.

Dessa forma, destaca-se a importância do acesso a informação de qualidade em relação ao produto, para que as mulheres possam vir a utilizar de forma adequada, sem abandonar o método regular e, principalmente, sem deixar de utilizar o preservativo, pois se trata do único método eficaz na prevenção de DST. É necessário que a contracepção de emergência apareça como suporte nas campanhas de prevenção de DST/ HIV, destacando-se que, somente no caso de rompimento do preservativo ou ato de violência sexual, deve ser associado o uso desse método evitando-se assim, uma gravidez não planejada.

\section{Colaboradores}

GM Alano e D Galato trabalharam na concepção, delineamento da pesquisa, redação e análise crítica do artigo. GM Alano, LN Costa, LR Miranda e D Galato trabalharam na pesquisa, análise, interpretação dos dados e redação do artigo. 


\section{Referências}

1. Wannmacher L. Anticoncepcionais Orais: O que há de novo. Uso racional de medicamento: temas selecionados. OPAS/OMS/MS. 2003; 1(1):1-6.

2. Rede brasileira de promoção de informação e disponibilização da contracepção de emergência. Contracepção de Emergência. [on line]. 2008 [acessado 2008 abr 18]. Disponível em: http://www.redece. org/mcce.htm\#importante

3. Wannmacher L. Contracepção de emergência: evidências versus preconceitos. Uso racional de medicamentos: temas selecionados. OPAS/OMS/MS. 2005; 2(6):1-6.

4. Brasil. Ministério da Saúde (MS). Secretaria de Atenção à Saúde. Departamento de Ações Programáticas Estratégicas. Área Técnica de Saúde da Mulher. Anticoncepção de emergência: perguntas e respostas para profissionais de saúde. Brasília: MS; 2005.

5. Costa NFP, Ferraz EA, Souza CT, Silva CFR, Almeida MG. Acesso à anticoncepção de emergência: velhas barreiras e novas questões. Rev Bras de Ginecol e Obstet 2008; 30(2):55-60.

6. Souza FG, Bona JC, Galato D. Comportamento de jovens de uma universidade do Sul do Brasil frente à prevenção de doenças sexualmente transmissíveis e gravidez. J bras Doenças Sex Transm 2007; 19(1):2229.

7. Associação Brasileira de Empresas de Pesquisas. Critério de classificação econômica Brasil. [on line]. 2008 [acessado 2008 jul 20]. Disponível em: http:// www.abep.org/codigosguias/Criterio_Brasil 2008.pdf

8. Lauritsen JM, Bruus M, Myatt MA. Programa para criar banco de dados. EpiData. Association Odense Denmark. [on line]. 2002 [acessado 2008 jul 22]. Disponível em: http://www.epidata.dk

9. Silveira PSP. Epi Info versão 6.0. [on line]. 2000 [acessado 2008 maio 18]. Disponível em: http:// www.dim.fm.usp.br/epiinfo/index.php\#_Toc 386598088

10. Taquette SR, Vilhena MM, Paula MC. Doenças sexualmente transmissíveis na adolescência: estudo de fatores de risco. Rev Soc Bras Med Trop 2004; 37(3):210-214.

11. Azevedo GE, Abdo CHN. Adolescentes de classe média do ensino fundamental: prática e conhecimento da sexualidade. Pediatria 2006; 28(3):184-190.

12. Borges ANV, Fujimori E, Hoga LAK, Contin MV. Práticas contraceptivas entre jovens universitários: o uso da anticoncepção de emergência Cad Saude Publica 2010; 26(4):816-826.
13. Payakachat N, Ragland D, Houston C. Impact of emergency contraception status on unintended pregnancy:observational data from a women's health practice. Pharmacy Practice 2010; 8(3):173-178.

14. Souza RA, Brandão ER. Marcos normativos da anticoncepção de emergência e as dificuldades de sua institucionalização nos serviços públicos de saúde. Rev Saude Coletiva 2009; 19(4):1067-1086.

15. Brasil. Ministério da Saúde (MS). Pesquisa Nacional de Demografia e Saúde da Criança e da Mulher 2006. Dimensões do processo reprodutivo e da saúde da criança. Série G. Brasília: MS; 2009.

16. Araújo MSP, Costa LOBF. Comportamento sexual e contracepção de emergência entre adolescentes de escolas públicas de Pernambuco, Brasil. Cad Saude Publica 2009; 25(3):551-562.

17. Figueiredo R. Uso de preservativos, risco e ocorrência de gravidez não planejada e conhecimento e acesso à contracepção de emergência entre mulheres com HIV/Aids. Cien Saude Colet 2010; 15(Supl. 1):1175-1183

18. Ros C, Miret M, Rue M. Estudio descriptivo sobre el uso dela anticoncepción de emergencia en Cataluña. Comparación entre uma zona rural y uma urbana. Gac Sanit 2009; 23(6):496-500.

19. Gainer E, Kenfack B, Mboudou E, Doh AS, Bouyer J. Menstrual bleeding patterns following levonorgestrel emergency contraception. Contraception 2006; 74(2):118-124

20. Bastos S, Bonfim JRA, Kalckmann, S, Regina Figueiredo R, Fernandes MEL. Prevenção de Doenças Sexualmente Transmissíveis e procura da contracepção de emergência em farmácias e drogarias do município de São Paulo. Saúde Soc 2009; 18(4):787-799.

Artigo apresentado em 08/06/2011

Aprovado em 18/07/2011

Versão final aprovada em 31/07/2011 